\title{
Model-based Leakage Detection in a Pulverized Coal Injection Vessel
}

\author{
Andreas Johansson and Alexander Medvedev \\ Control Engineering Group \\ Luleå University of Technology \\ SE-971 87 Luleå, Sweden
}

\begin{abstract}
A method for detecting and isolating incipient leakages in the valves of a pulverized coal injection vessel for a blast furnace process is presented. Non-linear physical gray-box models of the plant are developed. Values of the unknown parameters are estimated by identification. Observers are constructed for these models and the residuals are used in a Generalized Likelihood Ratio test. The method is successfully tested with real leakages intentionally introduced in the plant.
\end{abstract}

\section{INTRODUCTION}

Fault detection and isolation is a potentially powerful tool for achieving security and effective maintenance in various types of processes. Applications in aviation and automotive technology are common (see for example [1], [2]), but there are also many examples of fault detection in general industrial processes, like electrical motors and hydraulic systems [3], [4]. See [5] for a survey of recent simulations and implementations of fault detection systems.

The basic terminology and techniques for fault detection can be found in the survey by Frank [6]. State estimation by observers is often used. A number of different techniques exist, for example Unknown Input Observers, Dedicated Observers, Parity Space and Kalman Filter Methods. A survey on non-linear observers is given in [7].

The fundamental question in fault detection is whether the nominal non-faulty model should be accepted or rejected. This fact emphasizes the similarities to model validation. The detection of abrupt and incipient faults can thus be interpreted as local and global model validation, respectively.

This article concerns detection of incipient faults (leakages) in the valves of a pressurized coal injection vessel for a blast furnace process. The blast furnace is used in iron making to reduce ore into hot metal. The reduction agent is coal, in the form of coke or coal powder, of which the latter is less expensive. Coal powder can also be easier used to control the temperature of the blast furnace. The prime drawback of coal powder is its flammability. The ability to self-ignite in contact with air makes it inconvenient to transport. Any storage and transport has to take place under inert conditions and it has to be injected under pressure directly into the reaction core. For these reasons it is of interest to have a reliable coal powder injection plant. A leakage in a valve could for example make it possible for air to enter the injection vessel with possibly catastrophic consequences. In fact, one of the prime motivations for this work was a fire in one of the injection vessels at SSAB Tunnplät in Luleå.

Andreas.Johansson@sm.luth.se

Alexander.Medvedev@sm.luth.se

$0-7803-4530-4 / 98 \$ 10.00$ ๑ 1998 AACC

\section{A. The plant}

An overview of an injection vessel is shown in Fig. 1. In addition to the sensors in the figure, there is also a weighing equipment that measures the total mass of the content in the injection vessel. The injection process is cyclic and can be

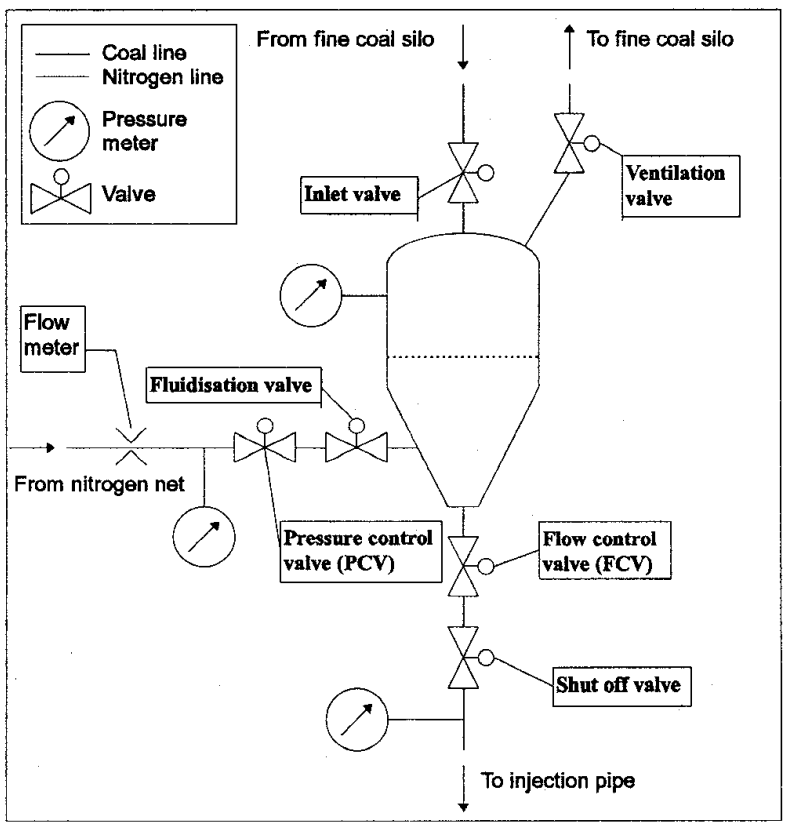

Fig. 1. The injection vessel

divided into the four phases shown in Table I.

\section{Modeling}

The notation used in the sequel is summarized in the appendix.

\section{A. Flow through Valves}

The flow through a valve is assumed to be the product of two factors. One, denoted as $f$ in (1), is a function of the pressures on each side of the valve, and the other, $k$, is a time-varying factor that is, for a control valve, a function of the input signal.

$$
q(t)=k(t) f\left(p_{1}(t), p_{2}(t)\right)
$$

The pressure $p_{1}$ is defined to be the pressure on the side that a positive-valued flow emerges from, while $p_{2}$ is the pressure on the opposite side. 
TABLE I

THE INJECTION CYCLE

\begin{tabular}{|l|l|l|}
\hline Phase & Action & Effect \\
\hline \hline Charging & Inlet valve is opened & $\begin{array}{l}\text { The vessel is filled } \\
\text { with coal powder }\end{array}$ \\
\hline $\begin{array}{l}\text { Pressuri- } \\
\text { zation }\end{array}$ & $\begin{array}{l}\text { Inlet and ventila- } \\
\text { tion valves are closed. } \\
\text { Fluidization and pres- } \\
\text { sure control valves } \\
\text { are opened }\end{array}$ & $\begin{array}{l}\text { The pressure rises } \\
\text { to 950 kPa }\end{array}$ \\
\hline Injection & $\begin{array}{l}\text { Flow control and } \\
\text { shutoff valves are } \\
\text { opened }\end{array}$ & $\begin{array}{l}\text { Coal powder in } \\
\text { injected into the } \\
\text { blast furnace }\end{array}$ \\
\hline Ventilation & $\begin{array}{l}\text { Ventilation valve is } \\
\text { opened. All other } \\
\text { valves are closed }\end{array}$ & $\begin{array}{l}\text { The vessel is de- } \\
\text { pressurized }\end{array}$ \\
\hline
\end{tabular}

For a control valve, the factor $k(t)$ in (1) is a function of the input signal $u$, i.e. $k(t)=k g(u(t))$ where $k$ is an unknown scaling factor and $g(u)$ is called the characteristic function of the valve. In order to make the definition above unambiguous it is also stated that $g(1)=1$.

\section{A.1 Pressure Control Valve (PCV)}

The flow of nitrogen through the PCV can be expressed as $q_{N, P}=k_{N, P} f_{\text {gas }}\left(p_{\mathcal{N}}, p\right) g_{P}\left(u_{P}\right)$ where $f_{\text {gas }}$ is a function describing the pressure dependency of the flow of gas through a valve and $g_{P}$ is the characteristic function of the PCV. Furthermore, $p_{\mathcal{N}}$ represents the pressure in the nitrogen net and $u_{P}$ is the input signal to the PCV.

\section{A.2 Flow Control Valve (FCV)}

Through the FCV there is a flow of both nitrogen and coal powder, i.e. a two component flow. To simplify the model, the two flows are assumed to be independent, and the coal powder is regarded as a liquid. More accurate models are available [8], but since the flow of coal powder is controlled and to be held constant, the accuracy of the physical model is not of crucial importance. Thus, the flow of coal powder is $q_{C, F}=k_{C, F} f_{\text {liq }}\left(p, p_{\mathcal{I}}\right) g_{F}\left(u_{F}\right)$ where $f_{\text {liq }}$ is a function describing the pressure-dependency of the flow of liquid through a valve and $g_{F}$ is the characteristic function of the FCV. Moreover, $p_{\mathcal{I}}$ is the pressure in the injection pipe and $u_{F}$ is the control signal for the FCV. The nitrogen flow is $q_{N, F}=k_{N, F} f_{\text {gas }}\left(p, p_{\mathcal{I}}\right) g_{F}\left(u_{F}\right)$

\section{A.3 Ventilation Valve (VV)}

In order to obtain a smooth flow in the ventilation valve, it is controlled by a hydraulic feedback. Since this control signal is not measured, the VV has to be modelled as a closed loop.

The flow through the VV goes to the fine coal silo, which can be considered to have infinite volume. This means that the pressure in the fine coal silo is constant. The flow through the $V V$ is thus a function of the pressure in the injection vessel only, i.e. $q_{N, V}=f_{N, V}(p)$. The function $f_{N, V}(p)$ is simply taken to be a polynomial of order J, i.e. $f_{N, V}(p)=$ $\sum_{j=0}^{J} k_{j} p^{j}$.
TABLE II

LEAKAGES

\begin{tabular}{|l|c|}
\hline Leakage & Notation \\
\hline \hline To the atmosphere & $\mathcal{A}$ \\
From the nitrogen net & $\mathcal{N}$ \\
To/from the injection pipe & $\mathcal{I}$ \\
No Leakage & $\emptyset$ \\
\hline
\end{tabular}

\section{A.4 Leakages}

Three different leakages are considered (Table II). The set of leakages is defined as $\mathcal{L} \triangleq\{\mathcal{A}, \mathcal{N}, \mathcal{I}, \emptyset\}$. A leakage can be looked upon as the flow through a valve with an unknown control signal. The nitrogen leakage flow can thus be represented by

$$
q_{\ell}=k_{\ell} f_{\ell}(\cdot) \quad \ell \in \mathcal{L}
$$

where $k_{\ell}$ is an unknown time-varying factor and $f_{\ell}(\cdot)$ is a function of the pressures on each side of the leakage. The trivial leakage function for the event of no leakage is $f_{\emptyset}=0$. The other leakage functions $\left(f_{\mathcal{A}}, f_{\mathcal{N}}\right.$ and $\left.f_{\mathcal{I}}\right)$ are developed from $f_{\text {gas }}$ by making certain assumptions on the pressures in the vessel, nitrogen net and injection pipe.

\section{B. Output transformation}

The pressure and total mass ( $p$ and $m$ ) of the vessel can be calculated from the masses of coal and nitrogen ( $m_{C}$ and $m_{N}$ ) using basic physical principles including the ideal gas law. With the definitions $y \triangleq\left[\begin{array}{ll}m & p\end{array}\right]^{T}$ and $x \triangleq\left[\begin{array}{ll}m_{C} & m_{N}\end{array}\right]^{T}$ the transformation can be expressed as

$$
y=h(x) \triangleq\left[\begin{array}{c}
m_{C}+m_{N} \\
m_{N} \frac{R_{N} T \rho_{C}}{V \rho_{C}-m_{C}}
\end{array}\right]
$$

Index $C$ in the equation above refers to pure coal and not coal powder. The reason for this is that the nitrogen is assumed to fill out the space between the coal particles. Since $h(x)$ is invertible, the coal and nitrogen masses can be considered measurable. The inverse transformation is given below.

$$
x=h^{-1}(y)=\left[\begin{array}{c}
\frac{m R_{N} T \rho_{C}-p V \rho_{C}}{R_{N} T \rho_{C}-p} \\
p \frac{V \rho_{C}-m}{R_{N} T \rho_{C}-p}
\end{array}\right]
$$

Upon entering the vessel, the nitrogen passes through the coal powder. Since nitrogen has much lower heat capacity than coal powder it is assumed to be momentarily heated to the temperature of the coal powder $\left(60-70^{\circ} \mathrm{C}\right)$. Therefore $T$ is given the constant value of $338 K$.

\section{Entire system}

The basis for the equations in this subsection is the assumption of mass conservation. The change in coal mass and nitrogen mass in the vessel is the sum of the material flows into the vessel.

\section{C.1 Pressurization and injection}

During the pressurization and injection phases the material transport takes place through the PCV and the FCV. 
Therefore, the change in the coal and nitrogen masses of the vessel can be expressed as

$$
\begin{aligned}
& \dot{m}_{C}=-q_{C, F} \\
& \dot{m}_{N}=-q_{N, F}+q_{N, P}
\end{aligned}
$$

where the $q$ 's denote the mass flows of coal (index $C$ ) and nitrogen (index $N$ ) through the FCV (index $F$ ) and the PCV (index $P$ ).

With the following definition

$$
u \triangleq\left[\begin{array}{l}
u_{C, F} \\
u_{N, F} \\
u_{N, P}
\end{array}\right] \triangleq\left[\begin{array}{l}
f_{\text {liq }}\left(p, p_{\mathcal{I}}\right) g_{F}\left(u_{F}\right) \\
f_{\text {gas }}\left(p, p_{\mathcal{I}}\right) g_{F}\left(u_{F}\right) \\
f_{\text {gas }}\left(p_{\mathcal{N}}, p\right) g_{P}\left(u_{P}\right)
\end{array}\right]
$$

the system (3) and (4) can be written as

$$
\dot{x}=A x+B u
$$

where

$$
A \triangleq\left[\begin{array}{cc}
a_{C} & 0 \\
0 & a_{N}
\end{array}\right] \quad B \triangleq\left[\begin{array}{ccc}
k_{C, F} & 0 & 0 \\
0 & -k_{N, F} & k_{N, P}
\end{array}\right]
$$

In principle, the variables $a_{C}$ and $a_{N}$ should be equal to zero, but in order to obtain extra degrees of freedom, they are considered to be unknown. When identifying, the parameter $a_{N}$ always takes a small negative value, which probably suggests inherent leakage in the vessel. The other parameter, $a_{C}$ takes on a small positive value, i.e. an unstable mode. However, since this instability is very small and not physically motivated, it does not present any problem.

Fig. 2 and Fig. 3 show a simulation of the entire non-linear system with input signals $u_{F}$ and $u_{P}$ and output signals $p$ and $m$.

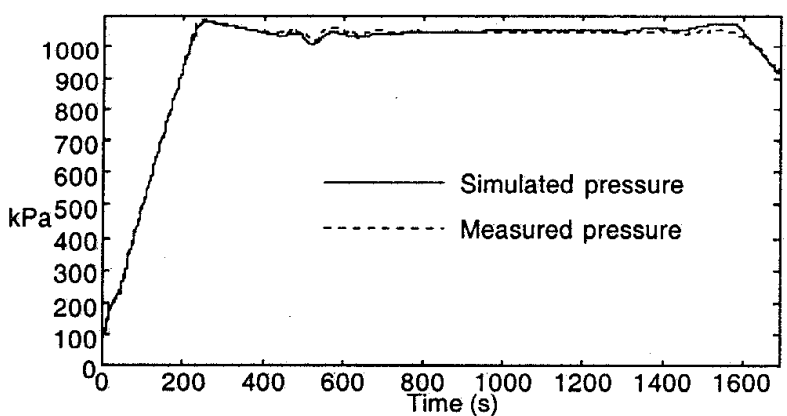

Fig. 2. Simulation of the pressure in the vessel during pressurization and injection

C.2 Ventilation

During the ventilation phase, the only material flow is the nitrogen flow through the ventilation valve. Therefore

$$
\begin{aligned}
& \dot{m}_{C}=0 \\
& \dot{m}_{N}=-q_{N, V}=-f_{N, V}(p)
\end{aligned}
$$

The nitrogen flow can be accurately described by a first order polynomial. This means that (8) can be written as

$$
\dot{m}_{N}=-k_{0}-k_{1} c\left(m_{C}\right) m_{N}
$$

where $c\left(m_{C}\right) \triangleq R_{N} T \rho_{C} /\left(V \rho_{C}-m_{C}\right)$. The coefficients $k_{0}$ and $k_{1}$ have been produced by least squares identification. Fig. 4 shows a simulation of the pressure during ventilation.

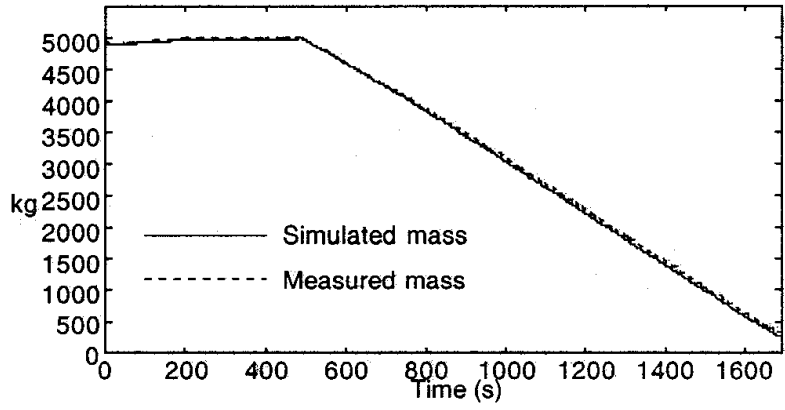

Fig. 3. Simulation of the mass in the vessel during pressurization and injection

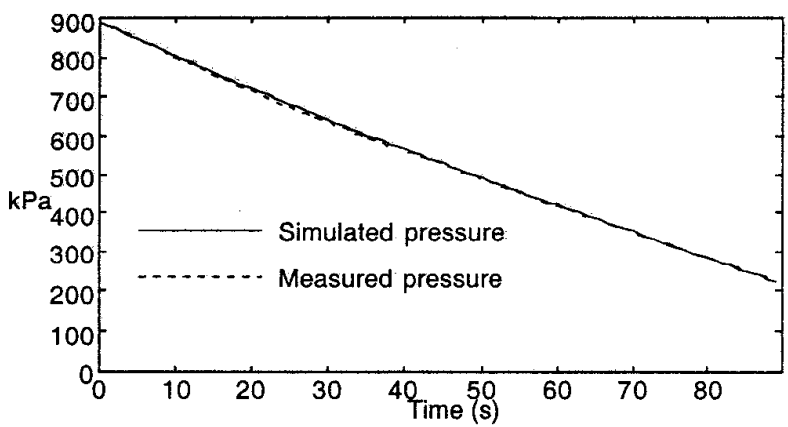

Fig. 4. Simulation of the pressure in the vessel during ventilation

\section{FAULT DETECTION}

The leakage flow is modeled as an extra term added to the right hand side of (4) and (8). The purpose of the fault detection algorithm is thus to calculate this term. However, the presence of noise makes it necessary to utilise feedback (i.e. observers) in order to obtain filtered estimates of the leakage flow. The nonlinear models developed in the previous section are of the Hammerstein type and thus linear observers can be used.

\section{A. Pressurization and injection}

When leakage is taken into account, equation (4) has to be extended with the term $q_{L}$, that represents the net leakage of nitrogen into the vessel, thus $\dot{m}_{N}=-q_{N, F}+q_{N, P}+q_{L}$. A linear observer for the system (5) is given by

$$
\dot{\hat{x}}=A \hat{x}+B u+K \epsilon
$$

where

$$
\epsilon \triangleq\left[\begin{array}{c}
\epsilon_{C} \\
\epsilon_{N}
\end{array}\right] \triangleq\left[\begin{array}{c}
m_{C}-\hat{m}_{C} \\
m_{N}-\hat{m}_{N}
\end{array}\right] \quad K \triangleq\left[\begin{array}{cc}
K_{C} & 0 \\
0 & K_{N}
\end{array}\right]
$$

Since in this system the state variables are decoupled (i.e. $A$ is diagonal), it is assumed that a diagonal observer gain matrix is sufficient. There is however a dependence between the state variables via the input- and output transformations and therefore the full-order observer is used. With the definitions above, it can be shown that the residual $\epsilon_{N}$ is the net leakage $q_{L}$, filtered through a first order filter. This filter is 
given by

$$
\epsilon_{N}(t)=\frac{1}{\mathbf{p}-a_{N}+K_{N}} q_{L}(t)=H_{\mathfrak{P}}(\mathbf{p}) q_{L}(t)
$$

where $\mathbf{p}$ is the differentiation operator. If the net leakage is assumed to be slowly varying in time, the residual divided by the static gain of the filter above is a good approximation of the net leakage corrupted by white noise.

$$
\bar{q}_{L}(t)=\frac{1}{H_{\mathfrak{p}}(0)} \epsilon_{N}(t)
$$

\section{B. Ventilation}

The net leakage $q_{L}$ is introduced in the ventilation system in a manner similar to the leakage in the pressurization and injection system i.e. $\dot{m}_{N}=-q_{N, V}+q_{L}$. An observer for the ventilation phase is given by

$$
\dot{\hat{m}}_{N}=-k_{0}-k_{1} c\left(m_{C}\right) \hat{m}_{N}+K_{N} \epsilon_{N}
$$

The residual $\epsilon_{N}$ is then the net leakage filtered through a first order filter.

$$
\epsilon_{N}(t)=\frac{1}{\mathbf{p}+k_{1} c\left(m_{C}\right)+K_{N}} q_{L}(t)=H_{\mathfrak{V}}(\mathbf{p}) q_{L}(t)
$$

As before, the residual is divided by the static gain of the filter.

$$
\bar{q}_{L}(t)=\frac{1}{H_{\mathfrak{V}}(0)} \epsilon_{N}(t)
$$

\section{ISOLATION OF LEAKAGES}

The development of the models and the observers in the previous sections is performed in continuous time to facilitate physical interpretation. However, since the systems are of the Hammerstein type, it is a straightforward task to discretize the observers. In the following, all signals are assumed to be discrete, which is indicated by the new time-variable $n$.

The calculated leakage is assumed to be the sum of a scaled leakage function and a disturbance, i.e. $\bar{q}_{L}(n)=k_{\ell} f_{\ell}(n)+$ $e(n)$ where $\ell \in \mathcal{L}$ and the term $e(n)$ is stationary zero-mean white Gaussian noise with variance $\sigma^{2}$, i.e. $e(n) \in \mathcal{N}(0, \sigma)$

Actually, $e(n)$ is not Gaussian (probably due to unmodelled nonlinearities), but this fact does not have any major influence on the results. When the transient behavior is excluded from the data, the residual is fairly near normal distribution, but the results are virtually the same

The factor $k_{\ell}$ in (2) is a measure for the size of the hole through which the leakage flow takes place. This means that $k_{\ell}$ varies slowly in time when considering incipient leakages. If it is assumed to be constant during a reasonably long period of time (for example a process cycle), it can be estimated using the Generalized Likelihood Ratio.

\section{A. Leakage hypothesis testing}

Four hypotheses $\left(\mathcal{H}_{\emptyset}, \mathcal{H}_{\mathcal{A}}, \mathcal{H}_{\mathcal{N}}\right.$ and $\left.\mathcal{H}_{\mathcal{I}}\right)$ are formed in agreement with the leakage events. The three leakage hypotheses are tested one by one against $\mathcal{H}_{\emptyset}$ using the Generalized Likelihood Ratio (GLR). If $\mathcal{H}_{\emptyset}$ is rejected in more than one of these tests, the hypothesis with the highest GLR is accepted. The likelihood functions for the hypotheses can be expressed as

$$
P_{\ell}\left(\bar{q}_{L}\right)=\prod_{n=1}^{N} \frac{1}{\sigma} \varphi\left(\frac{\bar{q}_{L}(n)-k_{\ell} f_{\ell}(n)}{\sigma}\right)
$$

where

$$
\varphi(\cdot)=\frac{1}{\sqrt{2 \pi}} e^{-\frac{(\cdot)^{2}}{2}}
$$

The GLR for each leakage hypothesis is

$$
\Lambda_{\ell}\left(\bar{q}_{L}\right)=\frac{\sup _{k_{\ell}>0} P_{\ell}\left(\bar{q}_{L}\right)}{P_{\emptyset}\left(\bar{q}_{L}\right)}
$$

The restriction on $k_{\ell}$ comes from the fact that a negative $k_{\ell}$ would imply a leakage flow from a lower pressure to a higher.

It can be shown that under the conditions above, the leakage likelihood function, $P_{\ell}\left(\bar{q}_{L}\right)$, is maximized by

$$
\hat{k}_{\ell}=\arg \sup _{k_{\ell}>0} P_{\ell}\left(\bar{q}_{L}\right)= \begin{cases}\frac{C_{\ell}}{\sum_{n=1}^{N} f_{\ell}^{2}(n)} & C_{\ell}>0 \\ 0 & \text { Otherwise }\end{cases}
$$

where $C_{\ell} \triangleq \sum_{n=1}^{N} \bar{q}_{L}(n) f_{\ell}(n)$ The logarithmic GLR can then be expressed as

$$
\ln \left(\Lambda_{\ell}\left(\bar{q}_{L}\right)\right)= \begin{cases}\frac{C_{\ell}^{2}}{2 \sigma^{2} \sum_{n=1}^{N} f_{\ell}^{2}(n)} & C_{\ell}>0 \\ 0 & \text { Otherwise }\end{cases}
$$

A threshold value, $\lambda$, for $\Lambda_{\ell}$ must be set. If $\Lambda_{\ell}$ exceeds this value then the null hypothesis, $\mathcal{H}_{\emptyset}$, is rejected. The threshold is generally calculated using the probability of rejecting the null hypothesis when it is true, i.e. $\mathbb{P}\left[\Lambda_{\ell}\left(\bar{q}_{L}\right)>\lambda\right] \triangleq \alpha$ where $\mathbb{P}$ is the probability operator. The probability $\alpha$ is called the level of the test and is usually set to a value in the range of $0,1 \% \leqslant \alpha \leqslant 5 \%$. In the case of the pressurization and injection system, however, the severity of the modelling errors causes the stationarity and zero-mean conditions on the disturbance $e(n)$ to fall. This makes it necessary to use very low levels to prevent a high rate of false alarms. For this reason, no test level is chosen but instead the threshold $\lambda$ is chosen on the basis of experimental data (Section V).

\section{Practical Results}

In order to validate the methods developed in this paper, experiments on the plant were carried out. Different leakages were artificially created during a period of six process cycles. The relevant signals were logged with a sampling time of $1 s$ and the net leakage was then calculated using (10) and (11)

The logarithmic GLR, $\ln \left(\Lambda_{\ell}\left(\bar{q}_{L}\right)\right)$, for each leakage type and each cycle is shown as diagrams in Fig. 5 and Fig. 6. The dashed line in Fig. 6 shows the threshold for GLR when $\alpha=$ $1 \%$ while the two dashed lines in Fig. 5 marks an interval for the threshold. Note that the hypothesis of leakage from the nitrogen net is absent in Fig. 5. The reason for this is that the pressure control valve, which connects the nitrogen net with the vessel, is open during pressurization and injection. There can for obvious reasons not be a leakage through an open valve.

Table III shows the conclusions that are drawn for the pressurization and injection phases when the threshold for the GLR is placed anywhere between the dashed lines of Fig. 5. Table IV shows the conclusions for the ventilation phase with level $1 \%$. Included in the tables are also the real leakages. It can be seen that the right conclusions are drawn in all cases but one. In this case there were two simultaneous leakages (Atmosphere and Nitrogen net) that nearly cancelled each other. 


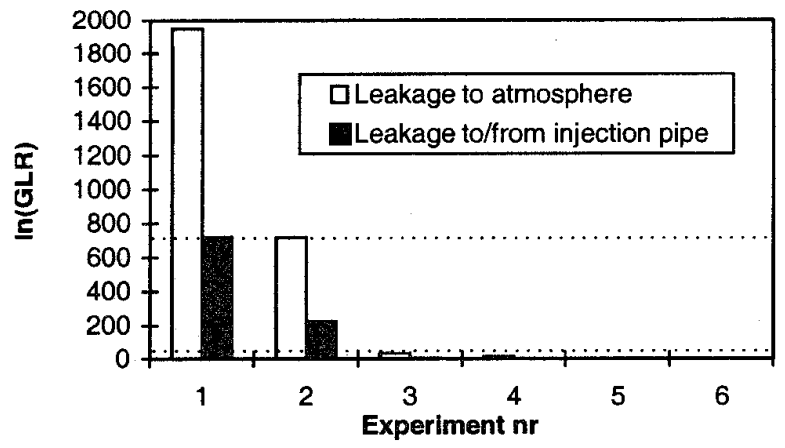

Fig. 5. The logarithm of the GLR for each leakage type and each experiment during pressurization and injection

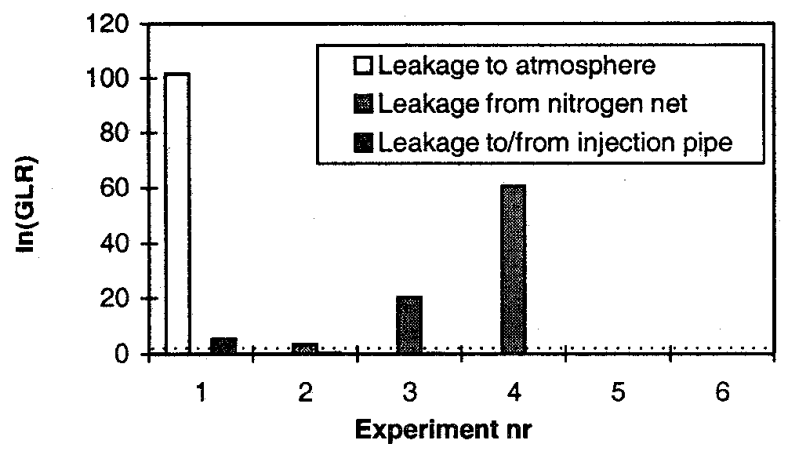

Fig. 6. The logarithm of the GLR for each leakage type and each experiment during ventilation

TABLE III

THE LEAKAGES DURING PRESSURIZATION AND INJECTION

\begin{tabular}{|c|l|l|}
\hline Cycle & Conclusion & Real Leakage \\
\hline \hline 1 & Atmosphere & Atmosphere \\
2 & Atmosphere & Atmosphere \\
3 & No leakage & No leakage \\
4 & No leakage & No leakage \\
5 & No leakage & No leakage \\
6 & No leakage & No leakage \\
\hline
\end{tabular}

TABLE IV

THE LEAKAGES DURING VENTILATION

\begin{tabular}{|c|c|c|}
\hline Cycle & Conclusion & Real Leakage \\
\hline 1 & Atmosphere & Atmosphere \\
\hline 2 & Nitrogen net & $\begin{array}{l}\text { Atmosphere and ni- } \\
\text { trogen net }\end{array}$ \\
\hline 3 & Nitrogen net & Nitrogen net \\
\hline 4 & Nitrogen net & Nitrogen net \\
\hline 5 & No leakage & No leakage \\
\hline 6 & No leakage & No leakage \\
\hline
\end{tabular}

\section{ConClusions}

It has been shown that model based fault detection is a possible way of detecting leakages in the valves of a pressurized system. A grey-box model for an injection vessel is developed and a method for detecting and isolating leakages is suggested. Experiments with leakages in different valves of the plant showed the effectiveness of the method.

\section{ACKNOWLEDGMENTS}

The authors want to thank the personnel of SSAB Tunnplåt in Luleå and in particular Robert Johansson, Ingemar Lundström and Bert Paavola for their time and effort.

\section{REFERENCES}

[1] F. Gustafsson, "A comparative study on change detection for some automotive applications," in European Control Conference, Brussels, Belgium, July 1997. CD-ROM.

[2] F. Gustafsson, "Slip-based tire-road friction estimation," Automatica, vol. 33 , no. 6 , pp. 1087-1099, 1997.

[3] J. Chai, R. Lyon, and J. Lang, "Non-invasive diagnostics of motoroperated valves," in Proceedings of the American Control Conference, vol. 2, pp. 2006-2012, 1997.

[4] W. Ge and C.-Z. Fang "Detection of faulty components via robust observation," International Journal of Control, vol. 47, no. 6, pp. $581-$ $599,1988$.

[5] R. Isermann and P. Ballé, "Trends in the application of model based fault detection and diagnosis of technical processes," in IFAC World Congress Proceedings, 1996. CD-ROM.

[6] P. M. Frank, "Fault diagnosis in dynamic systems using analytical and knowledge-based redundancy - a survey and some new results," Automatica, vol. 26 , no. 3 , pp. $459-474,1990$.

[7] E. A. Misawa and J. K. Hedrick, "Nonlinear observers - a state-ofthe-art survey," Journal of Dynamic Systems, Measurment and Control, vol. 111 , pp. 344-352, September 1989

[8] J. F. Davidson, R. Clift, and D. Harrison, eds., Fluidization. Academic Press, 1985.

[9] A. Bäckström and A. Johansson, "Fault detection in the pulverised coal injection," Master's thesis, Luleå University of Technology, 1997. (In Swedish).

[10] W. Birk and A. Medvedev, "Pressure and flow control of a pulverized coal injection vessel," in Proceedings of the 1997 IEEE international conferance on control applications, pp. 127-132, 1997.

\section{APPENDIX}

\section{NOMENCLATURE}

\begin{tabular}{|l|l|}
\hline Variable & Meaning \\
\hline \hline$a_{C}, a_{N}$ & $\begin{array}{l}\text { Parameters of the pressurizing and injec- } \\
\text { tion process }\end{array}$ \\
$k_{0}, k_{1}$ & Parameters of the ventilation process \\
$k_{C, F}, k_{N, F}$, & Parameters of the pressurizing and injec- \\
$k_{N, P}$ & tion process \\
$k_{\mathcal{A}}, k_{\mathcal{I}}, k_{\mathcal{N}}$ & Leakage parameters \\
$m$ & Total mass in the vessel \\
$m_{C}, m_{N}$ & Masses of coal and nitrogen in the vessel \\
$p$ & Pressure in the vessel \\
$p_{\mathcal{A}}, p_{\mathcal{I}}, p_{\mathcal{N}}$ & Pressure of the atmosphere, injection pipe \\
& and nitrogen net \\
$q_{C, F}$ & Mass flow of coal through the FCV \\
$q_{L}, \bar{q}_{L}$ & Real and approximated leakage flow \\
$q_{N, F}, q_{N, P}$, & Mass flow of nitrogen through the FCV \\
$q_{N, V}$ & PCV and VV \\
$R_{N}$ & Gas constant for nitrogen \\
$\rho_{C}$ & Density of coal \\
$T$ & Temperature in the vessel \\
$u_{F}, u_{P}$ & Control signal for the FCV and the PCV \\
$V$ & Volume of the vessel \\
\hline
\end{tabular}

